\title{
Huzinaga Projection Embedding for Efficient and Accurate Energies of Systems with Localized Spin-densities
}

\author{
Daniel S. Graham, ${ }^{\dagger}$ Xuelan Wen, ${ }^{\dagger}$ Dhabih V. Chulhai ${ }^{\dagger, \dagger}$ and Jason D. \\ Goodpaster*, $\uparrow$ \\ $\dagger$ Department of Chemistry, University of Minnesota. 207 Pleasant St. SE, Minneapolis \\ MN 55455, USA. \\ $\ddagger$ Current address: Department of Chemistry, University of Indianapolis. 1400 E Hanna \\ Ave., Indianapolis, IN 46227, USA. \\ E-mail: jgoodpas@umn.edu
}

\begin{abstract}
We demonstrate the accuracy and efficiency of the restricted open-shell and unrestricted formulation of the absolutely localized Huzinaga projection operator embedding method. Restricted open-shell and unrestricted Huzinaga projection embedding in the full system basis is formally exact to restricted open-shell and unrestricted KohnSham density functional theory, respectively. By utilizing the absolutely localized basis, we significantly improve the efficiency of the method, while maintaining high accuracy. Furthermore, the absolutely localized basis allows for high accuracy open-shell wave function methods to be embedded into a closed-shell density functional theory environment. The open-shell embedding method is shown to calculate electronic energies of a variety of systems to within $1 \mathrm{kcal} / \mathrm{mol}$ accuracy of the full system wave function result. For certain highly localized reactions, such as spin transition energies on
\end{abstract}


transition metals, we find that very few atoms are necessary to include in the wave function region, in order to achieve desired accuracy. This extension further broadens the applicability of our absolutely localized Huzinaga level-shift projection operator method to include open-shell species. Here we apply our method to several representative examples such as spin splitting energies, catalysis on transition metals, and radical reactions.

\section{Introduction}

The accurate simulation of systems with complex electronic character poses a significant challenge to modern computational methods. While Kohn-Sham density functional theory ${ }^{1,2}$ (KS-DFT) can provide accurate, computationally efficient results for many systems, current exchange-correlation functionals have significant limitations. It has been well documented that for reactions involving a transition metal ${ }^{3-6}$ and spin-state energetics of transition metal centers, ${ }^{7-13}$ the accuracy of KS-DFT is largely functional dependent with no obvious choice of which functional to use. Systems with degenerate and near-degenerate states, such as those with stretched covalent bonds and transition metals, are notoriously difficult to simulate accurately by modern KS-DFT methods. ${ }^{6,14-17}$

When studying systems with transition metals, correlated wave function (WF) methods such as coupled cluster (CC), and complete active space (CAS) are commonly considered more accurate. Unlike DFT, WF methods can be systematically improved (e.g. including additional excitations for CC calculations, or increasing the size of the CAS active space) to increase the accuracy of the calculation. However, WF calculations are often limited to a small model of the system of interest due to the high computational cost. This can be a successful strategy because of the localized nature of many reactions, but new errors may be introduced by dividing the full system into a smaller model and there is evidence that the process of choosing a model is not always a clear, systematically improvable process. ${ }^{18}$

Quantum embedding calculations seek to improve upon the small model simulations by 
including some influence of the full system on the final energy. Quantum embedding methods such as QM/MM, ${ }^{19}$ ONIOM,${ }^{20}$ DMET,${ }^{21,22}$ embedded mean-field theory, ${ }^{23-27}$ Green's function embedding, ${ }^{22,28-30}$ partition DFT, ${ }^{31-33}$ and DFT embedding ${ }^{22,34-37}$ among many others $^{38-43}$ were designed to combine the benefits of high accuracy and systematic improvability from WF theory for a small subsystem, while including effects from the full system at a comparably negligible computational cost. Projection operator based DFT embedding has been developed by many groups with significant success. ${ }^{44-55}$ Recent work in our group has focused on Huzinaga level-shift projection operator DFT embedding in the absolutely localized basis. We have demonstrated the efficiency, accuracy, and systematic improvability of this method for closed-shell molecular ground ${ }^{56,57}$ and excited states, ${ }^{18}$ and ground state properties of periodic systems. ${ }^{58}$ We have found that the absolutely localized basis not only reduces the size of the valence orbital space thus decreasing the computational cost of the WF calculation, it also improves the total accuracy of the reaction energy due to favorable error cancellation. ${ }^{57}$

Given our success with the Huzinaga based DFT embedding for closed-shell systems, we have expanded the method to allow restricted and unrestricted ground state embedded energies of molecular systems. We demonstrate highly accurate embedding energies for radical reactions, gas adsorption onto transition metals, and transition metal spin-state energetics. We also discuss how transition metal energies using the Huzinaga embedding method are far less functional dependent than traditional KS-DFT calculations.

\section{Theory}

We have developed unrestricted and restricted open-shell Huzinaga level-shift projection based embedding. Both methods are extensions of the closed-shell method described previously. ${ }^{57}$ In the sections that follow the full system is divided into two subsystems for simplicity, however the total system may be divided into any number of subsystems. 


\section{$2.1 \quad$ Unrestricted}

For unrestricted embedding, the total system electron density is divided into $\alpha$ and $\beta$ spin components, $\gamma_{\alpha}, \gamma_{\beta}$ and those components are then subdivided into subsystem $\mathrm{A}, \gamma^{\mathrm{A}}$, and subsystem B, $\gamma^{\mathrm{B}}$ where,

$$
\begin{aligned}
& \gamma_{\alpha}=\gamma_{\alpha}^{\mathrm{A}}+\gamma_{\alpha}^{\mathrm{B}}, \\
& \gamma^{\mathrm{A}}=\gamma_{\alpha}^{\mathrm{A}}+\gamma_{\beta}^{\mathrm{A}} .
\end{aligned}
$$

The definition of $\gamma_{\beta}$ is analogous to equation 1 and $\gamma^{\mathrm{B}}$ is analogous to equation 2. These subsystem spin electron densities are defined in the absolutely localized basis. The absolutely localized basis defines the Kohn-Sham orbitals in a subsystem using only those basis functions centered on atoms within that subsystem. In the absolutely localized basis, the $\alpha$ spin KohnSham orbitals of subsystem A are defined as,

$$
\phi_{i \alpha}^{\mathrm{A}}=\sum_{\mu} C_{i \mu \alpha}^{\mathrm{A}} \chi_{\mu}^{\mathrm{A}},
$$

where $\phi_{i \alpha}^{\mathrm{A}}$ are the $\alpha$ spin Kohn-Sham orbitals of subsystem A, $C_{i \mu \alpha}^{\mathrm{A}}$ are the spin orbital coefficients of subsystem A, and $\chi_{\mu}^{\mathrm{A}}$ are basis functions associated with subsystem A. This basis is also used for all embedded WF calculations in this paper.

In order to maintain orthogonality between subsystems, the unrestricted spin Huzinaga level-shift projection operator is added to the subsystem Hamiltonian. The unrestricted spin Huzinaga level-shift projection operator may be added to a subsystem Hamiltonian, allowing simple incorporation with any existing QM method utilizing an electron potential Hamiltonian. The form of the spin Huzinaga level-shift projection operator for subsystem A in the presence of subsystem B is,

$$
\mathbf{P}_{\alpha}^{\mathrm{B}}=-\left(\mathbf{F}_{\alpha}^{\mathrm{AB}} \gamma_{\alpha}^{\mathrm{B}} \mathbf{S}^{\mathrm{BA}}+\mathbf{S}^{\mathrm{AB}} \gamma_{\alpha}^{\mathrm{B}} \mathbf{F}_{\alpha}^{\mathrm{BA}}\right)
$$


where,

$$
\mathbf{F}_{\alpha}^{\mathrm{AB}}=\left\langle\chi^{\mathrm{A}}\left|\hat{F}_{\alpha}\right| \chi^{\mathrm{B}}\right\rangle
$$

with $\hat{F}_{\alpha}$ being the full system $\alpha$ spin Fock operators and,

$$
\mathbf{S}^{\mathrm{AB}}=\left\langle\chi^{\mathrm{A}} \mid \chi^{\mathrm{B}}\right\rangle
$$

Including the unrestricted spin Huzinaga level-shift projection operator into the spin Fock matrix of subsystem A embedded into subsystem B results in the projected $\alpha$ spin Fock matrix,

$$
\mathbf{f}_{\alpha}^{\mathrm{A}-\mathrm{in}-\mathrm{B}}=\mathbf{h}_{\alpha}^{\mathrm{A}-\mathrm{in}-\mathrm{B}}\left[\gamma_{\alpha}^{\mathrm{A}}, \gamma_{\beta}^{\mathrm{A}}, \gamma_{\alpha}^{\mathrm{B}}, \gamma_{\beta}^{\mathrm{B}}\right]+\mathbf{J}\left[\gamma^{\mathrm{A}}\right]+\mathbf{v}_{\mathrm{xc}}\left[\gamma_{\alpha}^{\mathrm{A}}, \gamma_{\beta}^{\mathrm{A}}\right]
$$

where $\mathbf{J}$ is the electron Coulomb potential, $\mathbf{v}_{\mathrm{xc}}$ is the exchange-correlation (XC) potential, and the embedded core Hamiltonian is

$$
\mathbf{h}_{\alpha}^{\mathrm{A}-\mathrm{in}-\mathrm{B}}\left[\gamma_{\alpha}^{\mathrm{A}}, \gamma_{\beta}^{\mathrm{A}}, \gamma_{\alpha}^{\mathrm{B}}, \gamma_{\beta}^{\mathrm{B}}\right]=\mathbf{h}+\mathbf{J}\left[\gamma^{\mathrm{A}}+\gamma^{\mathrm{B}}\right]-\mathbf{J}\left[\gamma^{\mathrm{A}}\right]+\mathbf{v}_{\mathrm{xc}}\left[\gamma_{\alpha}, \gamma_{\beta}\right]-\mathbf{v}_{\mathrm{xc}}\left[\gamma_{\alpha}^{\mathrm{A}}, \gamma_{\beta}^{\mathrm{A}}\right]+\mathbf{P}_{\alpha}^{\mathrm{B}}\left[\gamma_{\alpha}^{\mathrm{B}}\right]
$$

where $\mathbf{h}$ is the total one-electron Hamiltonian.

The embedded DFT energy of subsystem A, $E_{\mathrm{DFT}}^{\mathrm{A}}$, is calculated by,

$E_{\mathrm{DFT}}^{\mathrm{A}}=\operatorname{Tr}\left(\gamma_{\alpha}^{\mathrm{A}} \cdot \mathbf{h}_{\alpha}^{\mathrm{A}-\mathrm{in}-\mathrm{B}}\left[\gamma_{\alpha}^{\mathrm{A}}, \gamma_{\beta}^{\mathrm{A}}, \gamma_{\alpha}^{\mathrm{B}}, \gamma_{\beta}^{\mathrm{B}}\right]\right)+\operatorname{Tr}\left(\gamma_{\beta}^{\mathrm{A}} \cdot \mathbf{h}_{\beta}^{\mathrm{A}-\mathrm{in}-\mathrm{B}}\left[\gamma_{\alpha}^{\mathrm{A}}, \gamma_{\beta}^{\mathrm{A}}, \gamma_{\alpha}^{\mathrm{B}}, \gamma_{\beta}^{\mathrm{B}}\right]\right)+\mathrm{J}\left[\gamma^{\mathrm{A}}\right]+E_{\mathrm{xc}}\left[\gamma_{\alpha}^{\mathrm{A}}, \gamma_{\beta}^{\mathrm{A}}\right]$

and the WF energy of subsystem A embedded in the DFT potential of the full system, $E_{\mathrm{WF}}^{\mathrm{A}}$, is

$$
E_{\mathrm{WF}}^{\mathrm{A}}=\left\langle\Psi_{\alpha}^{\mathrm{A}}\left|\hat{H}_{\alpha}^{\mathrm{A}-\mathrm{in}-\mathrm{B}}\right| \Psi_{\alpha}^{\mathrm{A}}\right\rangle+\left\langle\Psi_{\beta}^{\mathrm{A}}\left|\hat{H}_{\beta}^{\mathrm{A}-\mathrm{in}-\mathrm{B}}\right| \Psi_{\beta}^{\mathrm{A}}\right\rangle
$$

Here $\hat{H}_{\alpha}^{\mathrm{A}-\mathrm{in}-\mathrm{B}}$ is

$$
\hat{H}_{\alpha}^{\mathrm{A}-\mathrm{in}-\mathrm{B}}=\hat{h}_{\alpha}^{\mathrm{A}-\mathrm{in}-\mathrm{B}}+\hat{g}_{\alpha}^{\mathrm{A}}
$$

where $\hat{h}_{\alpha}^{\mathrm{A}-\mathrm{in}-\mathrm{B}}$ is equivalent to equation 8 (now written in operator form) and $\hat{g}_{\alpha}^{\mathrm{A}}$ is the $\alpha$ two- 
electron operator for a given WF theory acting on the electrons of subsystem A. Therefore the overall WF-in-DFT embedding energy is,

$$
E_{\mathrm{WF}-\mathrm{in}-\mathrm{DFT}}^{\text {Full }}=E_{\mathrm{KS}-\mathrm{DFT}}^{\mathrm{Full}}-E_{\mathrm{DFT}}^{\mathrm{A}}+E_{\mathrm{WF}}^{\mathrm{A}}
$$

and uses an subtractive embedding framework like ONIOM, ${ }^{20}$ where $E_{\text {WF-in-DFT }}^{\text {Full }}$ is the total WF-in-DFT energy, $E_{\mathrm{KS}-\mathrm{DFT}}^{\text {Full }}$ is the canonical KS-DFT of the full system, $E_{\mathrm{DFT}}^{\mathrm{A}}$ is the DFT energy of subsystem A embedded in the full system.

\section{$2.2 \quad$ Restricted Open-shell}

For restricted open-shell embedding, the total system electron density may be divided as in equations 1 and 2. The same absolutely localized basis is also employed, however defined in the restricted sense as,

$$
\phi_{i}^{\mathrm{A}}=\sum_{\mu} C_{i \mu}^{\mathrm{A}} \chi_{\mu}^{\mathrm{A}}
$$

where $\phi_{i}^{\mathrm{A}}$ are the restricted open-shell Kohn-Sham orbitals of subsystem $\mathrm{A}, C_{i \mu}^{\mathrm{A}}$ are the

restricted open-shell orbital coefficients of subsystem A, and $\chi_{\mu}^{\mathrm{A}}$ are basis functions associated with subsystem A. The restricted open-shell Huzinaga level-shift projection operator is defined using the restricted open-shell Fock matrix, $\mathbf{F}_{\mathrm{RO}}$,

$$
\mathbf{P}^{\mathrm{B}}=-\frac{1}{2}\left(\mathbf{F}_{\mathrm{RO}}^{\mathrm{AB}} \gamma^{\mathrm{B}} \mathbf{S}^{\mathrm{BA}}+\mathbf{S}^{\mathrm{AB}} \gamma^{\mathrm{B}} \mathbf{F}_{\mathrm{RO}}^{\mathrm{BA}}\right)
$$

Here, the restricted open-shell Fock matrix is,

$$
\mathbf{F}_{\mathrm{RO}}=\left(\begin{array}{ccc}
\mathbf{F}_{c c}^{\mathrm{cs}} & \mathbf{F}_{c o}^{\beta} & \mathbf{F}_{c v}^{\mathrm{cs}} \\
\mathbf{F}_{o c}^{\beta} & \mathbf{F}_{o o}^{\mathrm{cs}} & \mathbf{F}_{o v}^{\alpha} \\
\mathbf{F}_{v c}^{\mathrm{cs}} & \mathbf{F}_{v o}^{\alpha} & \mathbf{F}_{v v}^{c s}
\end{array}\right) \quad \begin{gathered}
\operatorname{core}(c) \\
\operatorname{open-shell}(o) \\
\operatorname{virtual}(v)
\end{gathered}
$$


where $\mathbf{F}^{\alpha}$ and $\mathbf{F}^{\beta}$ are the $\alpha$ and $\beta$ spin Fock matrices respectively, and $\mathbf{F}^{\mathrm{cs}}$ is $\left(\mathbf{F}^{\alpha}+\mathbf{F}^{\beta}\right) / 2$. Then we arrive at $\mathbf{F}_{\mathrm{RO}}^{\mathrm{AB}}$ as,

$$
\mathbf{F}_{\mathrm{RO}}^{\mathrm{AB}}=\left\langle\chi^{\mathrm{A}}\left|\hat{F}_{\mathrm{RO}}\right| \chi^{\mathrm{B}}\right\rangle
$$

The restricted open-shell embedded core Hamiltonian is,

$$
\mathbf{h}_{\mathrm{RO}}^{\mathrm{A}-\mathrm{in}-\mathrm{B}}\left[\gamma_{\alpha}^{\mathrm{A}}, \gamma_{\beta}^{\mathrm{A}}, \gamma_{\alpha}^{\mathrm{B}}, \gamma_{\beta}^{\mathrm{B}}\right]=\mathbf{h}+\mathbf{J}\left[\gamma^{\mathrm{A}}+\gamma^{\mathrm{B}}\right]-\mathbf{J}\left[\gamma^{\mathrm{A}}\right]+\mathbf{v}_{\mathrm{xc}, \operatorname{RO}}\left[\gamma_{\alpha}, \gamma_{\beta}\right]-\mathbf{v}_{\mathrm{xc}}, \operatorname{RO}\left[\gamma_{\alpha}^{\mathrm{A}}, \gamma_{\beta}^{\mathrm{A}}\right]+\mathbf{P}^{\mathrm{B}}\left[\gamma^{\mathrm{B}}\right]
$$

where $\mathbf{v}_{\mathrm{xc}}$, RO is the restricted open-shell exchange-correlation potential matrix defined as

$$
\mathbf{v}_{\mathrm{xc}, \operatorname{RO}}\left[\gamma_{\alpha}, \gamma_{\beta}\right]=\left(\begin{array}{ccc}
\mathbf{v}_{\mathrm{xc}}^{\mathrm{cs}}\left[\gamma_{\alpha}, \gamma_{\beta}\right]_{c c} & \mathbf{v}_{\mathrm{xc}}\left[\gamma_{\beta}\right]_{c o} & \mathbf{v}_{\mathrm{xc}}^{\mathrm{cs}}\left[\gamma_{\alpha}, \gamma_{\beta}\right]_{c v} \\
\mathbf{v}_{\mathrm{xc}}\left[\gamma_{\beta}\right]_{o c} & \mathbf{v}_{\mathrm{xc}}^{\mathrm{cs}}\left[\gamma_{\alpha}, \gamma_{\beta}\right]_{o o} & \mathbf{v}_{\mathrm{xc}}\left[\gamma_{\alpha}\right]_{o v} \\
\mathbf{v}_{\mathrm{xc}}^{\mathrm{cs}}\left[\gamma_{\alpha}, \gamma_{\beta}\right]_{v c} & \mathbf{v}_{\mathrm{xc}}\left[\gamma_{\alpha}\right]_{v o} & \mathbf{v}_{\mathrm{xc}}^{\mathrm{cs}}\left[\gamma_{\alpha}, \gamma_{\beta}\right]_{v v}
\end{array}\right) \quad \text { open-shell }(o)
$$

with

$$
\mathbf{v}_{\mathrm{xc}}^{\mathrm{cs}}\left[\gamma_{\alpha}, \gamma_{\beta}\right]=\frac{\mathbf{v}_{\mathrm{xc}}\left[\gamma_{\alpha}\right]+\mathbf{v}_{\mathrm{xc}}\left[\gamma_{\beta}\right]}{2}
$$

The embedded DFT energy of subsystem $\mathrm{A}, E_{\mathrm{DFT}}^{\mathrm{A}}$, is calculated by,

$$
E_{\mathrm{DFT}}^{\mathrm{A}}=\operatorname{Tr}\left(\gamma^{\mathrm{A}} \cdot \mathbf{h}_{\mathrm{RO}}^{\mathrm{A}-\mathrm{in}-\mathrm{B}}\left[\gamma_{\alpha}^{\mathrm{A}}, \gamma_{\alpha}^{\mathrm{B}} \gamma_{\beta}^{\mathrm{A}}, \gamma_{\beta}^{\mathrm{B}}\right]\right)+\mathrm{J}\left[\gamma^{\mathrm{A}}\right]+E_{\mathrm{xc}}\left[\gamma_{\alpha}^{\mathrm{A}}\right]+E_{\mathrm{xc}}\left[\gamma_{\beta}^{\mathrm{A}}\right]
$$

and the WF energy of subsystem A embedded in the DFT potential of the full system, $E_{\mathrm{WF}}^{\mathrm{A}}$, is

$$
E_{\mathrm{WF}}^{\mathrm{A}}=\left\langle\Psi^{\mathrm{A}}\left|\hat{H}_{\mathrm{RO}}^{\mathrm{A}-\mathrm{in}-\mathrm{B}}\right| \Psi^{\mathrm{A}}\right\rangle .
$$

Here $\hat{H}_{\mathrm{RO}}^{\mathrm{A}-\mathrm{in}-\mathrm{B}}$ is

$$
\hat{H}_{\mathrm{RO}}^{\mathrm{A}-\mathrm{in}-\mathrm{B}}=\hat{h}_{\mathrm{RO}}^{\mathrm{A}-\mathrm{in}-\mathrm{B}}+\hat{g}_{\mathrm{RO}}^{\mathrm{A}}
$$

where $\hat{h}_{\mathrm{RO}}^{\mathrm{A}-\mathrm{in}-\mathrm{B}}$ is equivalent to equation 17 (now written in operator form) and $\hat{g}_{\mathrm{RO}}^{\mathrm{A}}$ is the restricted open-shell two-electron operator for a given restricted open-shell WF theory act- 
ing on the electrons of subsystem A. The total WF-in-DFT embedding is the same as for unrestricted embedding, equation 12. We note that for both unrestricted and restricted open-shell embedding, if the full system basis is employed the exact KS-DFT energy and density would be obtained from the DFT-in-DFT embedding procedure as this formulation is formally exact.

\section{Computational Details}

The thiol-ene reaction molecular geometries were optimized using Gaussian $16^{59}$ using the M06 functional ${ }^{60}$ and aug-cc-pVDZ basis. ${ }^{61}$ The Fe-MOF-74 cluster geometry was calculated using Gaussian $16^{59}$ following the procedure outlined by Lee and coworkers. ${ }^{62}$ Fe spin transition cluster geometries originate from a recent study by Radon. ${ }^{63}$ Furthermore, all geometries are reported in the Supporting Information (SI). Single point DFT and UCCSD(T) energy calculations were performed using PySCF version 1.7.4 ${ }^{64}$ and all ROCCSD(T) and CASPT2 calculations were done using Molpro 2019.2. ${ }^{65-71}$ WF-in-DFT embedding energies were calculated using our Quantum Solid-state and Molecular Embedding code ${ }^{72}$ (QSoME) via the same freeze-and-thaw scheme outlined in our previous paper. ${ }^{57}$ All relevant output files including full system energies are included in the SI. Interestingly, we have found that embedding two charged subsystems results in higher accuracy than embedding two openshell subsystems. This result supports our previous findings and further details are provided in the SI.

\section{Results and Discussion}

With the addition of restricted open-shell and unrestricted embedding, a myriad of new chemical systems may be studied using our absolutely localized Huzinaga embedding method. Here we have chosen several open-shell systems as demonstrative examples of the broad applicability of our embedding method. 


\subsection{Radical Reactions}

Systematic improvability, that is where energies improve through a well defined process, is an important feature of our embedding method. We have demonstrated previously that the method is systematically improvable for closed-shell systems, ${ }^{57}$ and here have chosen to study a simple radical thiol-ene reaction (Fig. 1) to demonstrate the same systematic improvability for our open-shell embedding method. The system is divided into a series of incrementally larger WF subsystems in order to understand how the energies changed as the size of the WF region grows. For this calculation, we chose $\operatorname{CCSD}(\mathrm{T})$ for the WF method and used the M06 DFT exchange-functional. We calculated the reaction energy using

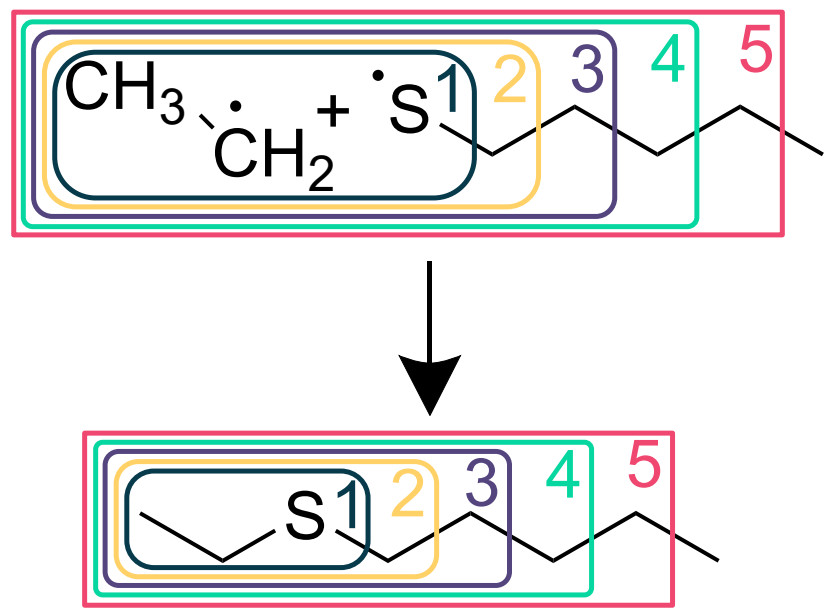

Figure 1: 1-(Ethylsulfyl)pentane formation by thiol-ene radical reaction. Numbering indicates the size of the WF subsystem. Implied hydrogen are included within the subsystem containing their associated bonded carbon.

both restricted open-shell and unrestricted Huzinaga embedding and compared the result to the full system $\operatorname{CCSD}(\mathrm{T})$ reaction energy. As is evident from Fig. 2, our embedding method exhibits desirable systematic improvability with increasing WF subsystem size for restricted and unrestricted calculations. Furthermore, including only those atoms directly bonded to the reaction center is sufficient to recreate full system $\operatorname{CCSD}(\mathrm{T})$ results to within $1 \mathrm{kcal} / \mathrm{mol}$, demonstrated by the high accuracy for WF subsystem size 2. A significant difference between $\operatorname{CCSD}(\mathrm{T})$ and embedding results for WF subsystem size 1 was expected based on our previous closed-shell embedding calculations. ${ }^{57}$ Additionally, the difference 
between $\operatorname{CCSD}(\mathrm{T})$ and embedding results does not monotonically converge to exactly zero due to our use of the absolutely localized basis, however the difference does converge to well within accepted chemical accuracy $(1 \mathrm{kcal} / \mathrm{mol})$.

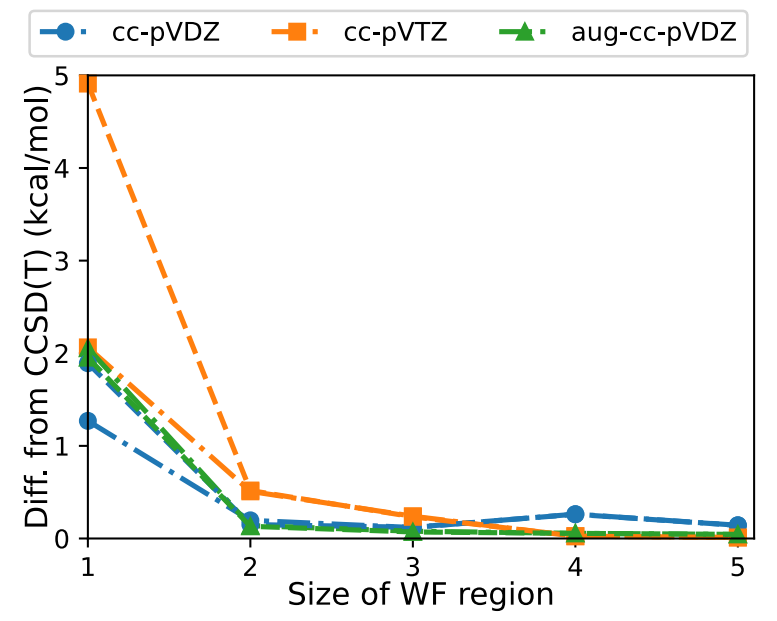

Figure 2: Absolute energy difference of $\operatorname{CCSD}(\mathrm{T})$ embedded in M06 for 1(Ethylsulfyl)pentane formation by thiol-ene radical reaction compared to full system $\operatorname{CCSD}(\mathrm{T})$. Subsystem size divisions correspond with the division in Fig. 1. Dashed lines represent unrestricted embedding, dash dotted lines represent restricted open-shell embedding.

\subsection{Fe-MOF-74 Gas Adsorption}

As was mentioned in the introduction, transition metal systems are notoriously difficult for DFT methods to calculate accurately. We have successfully calculated the energy of hydrogen gas adsorption on an closed shell singlet Fe-MOF-74 cluster model using our closed-shell method. ${ }^{57}$ However, the ground state spin for this model is actually a quintet. Therefore, we now calculate the adsorption energy of the true ground electronic state. We divided the system following the scheme shown in Fig. 3 and embedded CASPT2 subsystems within M06 DFT. All five Fe 3d orbitals with seven electrons, and both adsorbate hydrogen 1s orbitals with two electrons were included as the active space for the CASPT2 calculation. Our results, shown in Fig. 4, once again demonstrate highly accurate WF results once all atoms adjacent to the reaction are included in the WF subsystem (subsystem size 3). Here 


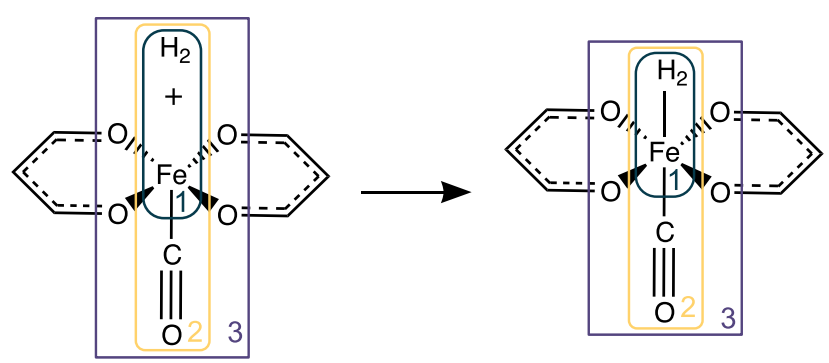

Figure 3: Hydrogen gas adsorption reaction on a Fe-MOF-74 cluster model. WF subsystem divisions are specified; the smallest subsystem includes only the Fe and hydrogen adsorbate.

for the first time we see an increase in error as the size of the WF subsystem is increased however, it is important to note that this error increase only occurs for restricted openshell embedding. We attribute this exception to the smooth convergence of the embedding energy to WF results to the restricted open-shell orbital restriction in conjunction with the absolutely localized restriction. In our restricted open-shell embedding, the unpaired electron must be localized to the atoms in the WF subsystem while the DFT subsystem is described as a closed shell singlet because of our absolutely localized basis. This can result in increased errors due to forced localization of the spin density if the WF region is too small. We do note that there is a uniform convergence once the WF subsystem size is sufficiently large.

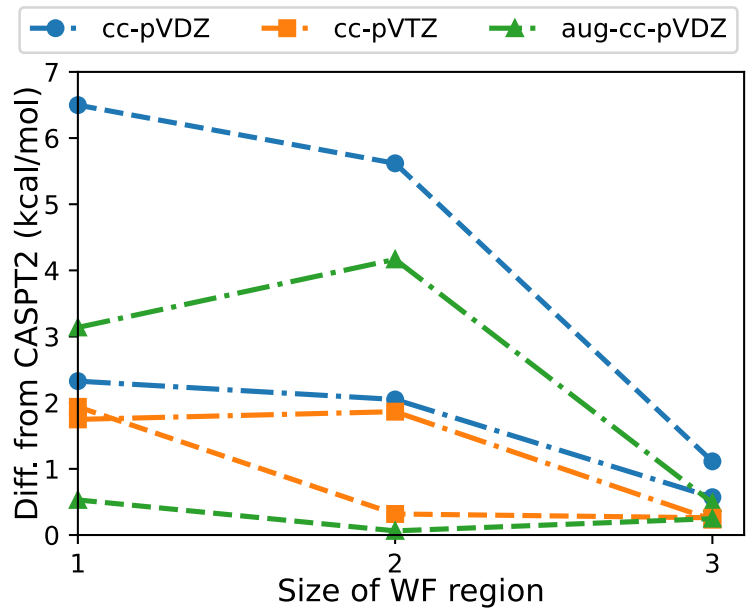

Figure 4: Absolute energy difference of CASPT2 embedded in M06 DFT hydrogen adsorption compared to full cluster CASPT2 results. Dashed lines represent unrestricted embedding, dash dotted lines represent restricted open-shell embedding. 


\subsection{Spin Transition Energy}

One particularly difficult property to accurately calculate for many systems is the spintransition energy (STE), or vertical excitation energy. Spin-transition energies for systems with transition metals often have a significant dependency on the exchange-correlation functional. ${ }^{73}$ The spin density is typically localized to the transition metal which makes spintransition energy calculations an appealing target for embedding methods. To demonstrate the effectiveness of absolutely localized Huzinaga WF-in-DFT embedding, we calculated the STEs of two Fe cluster systems previously identified by Radon as having high functional dependence. ${ }^{63}$ We compared UCCSD(T) calculations on the full system to embedding STEs using a variety of functionals.

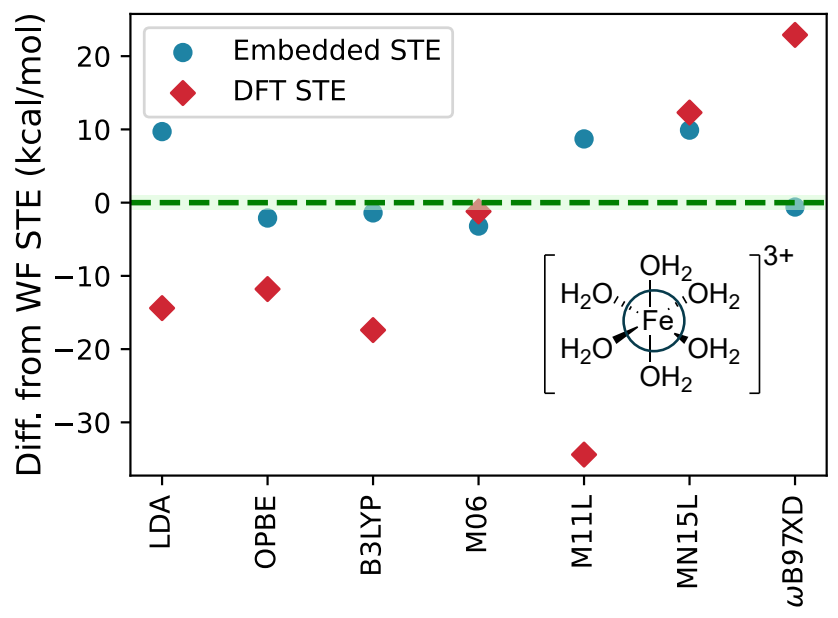

Figure 5: Spin-transition energy (STE) difference of embedding in a variety of DFT XC functionals compared to $\operatorname{UCCSD}(\mathrm{T})$. Green shaded region indicates $1 \mathrm{kcal} / \mathrm{mol}$ difference from full system WF results.

For the smaller, weak field ligand system shown in Fig. 5, the embedded WF region is limited to only include the Fe (any larger would contain the full system). There still exists some exchange-correlation functional dependency, but embedding reduces the deviation from more than $50 \mathrm{kcal} / \mathrm{mol}$ between functionals to less than $10 \mathrm{kcal} / \mathrm{mol}$. Additionally, for all functionals except the M06 functional embedding improves upon the DFT results. In the case of the M06 functional, we believe the slight increase in error by $2 \mathrm{kcal} / \mathrm{mol}$ is due to the fact that the M06 functional closely recreates the WF results on its own and embedding 


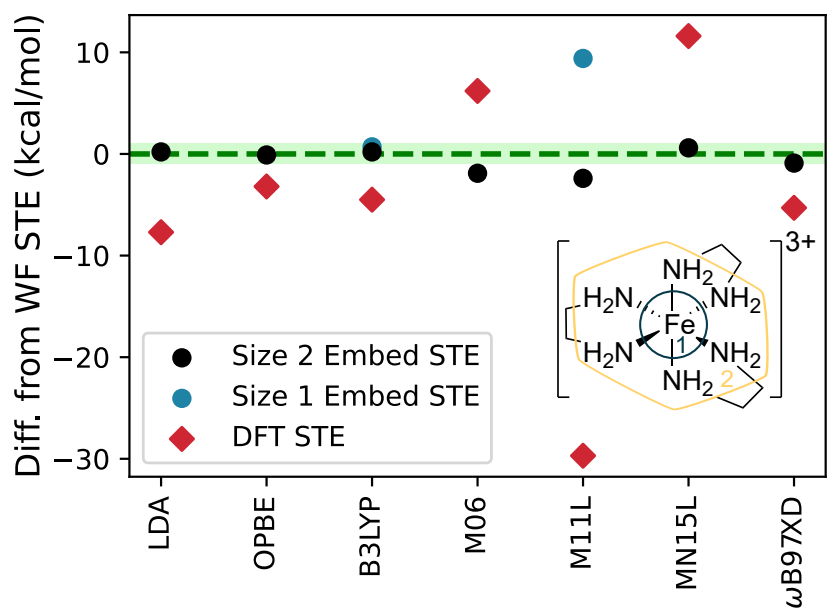

Figure 6: Spin-transition energy (STE) difference of embedding in a variety of DFT XC functionals compared to $\mathrm{UCCSD}(\mathrm{T})$. Green shaded region indicates $1 \mathrm{kcal} / \mathrm{mol}$ difference from full system WF results. The only subsystem size 1 embedding results presented are for B3LYP and M11L functionals as all other functionals failed to converge for subsystem size 1 .

introduces minor errors for small subsystem divisions as we have seen previously. Some embedding error for small WF subsystem sizes is expected however, and has been well documented by Bensberg and Neugebauer in a recent article ${ }^{55}$

When looking at the strong field system shown in Fig. 6, it is clear that expanding the WF region does significantly improve the embedding results and in every case more closely recreates the $\mathrm{UCCSD}(\mathrm{T})$ results than the corresponding $\mathrm{XC}$ functional. This system proved difficult to converge for subsystem size 1, however following our general recommendation of including atoms adjacent to the region of chemical interest results in spin transition energies that are in close agreement with $\mathrm{UCCSD}(\mathrm{T})$ results for the full system, regardless of $\mathrm{XC}$ functional.

\section{Conclusions}

With this improved Huzinaga embedding method, we have demonstrated the effectiveness of the method for nearly every domain of Born-Oppenheimer localized chemical reaction: periodic or non-periodic, ground state and excited state, closed-shell or open-shell. Specifi- 
cally here we have shown that our method can accurately recreate WF results for a variety of open-shell systems embedding a variety of QM methods. These WF results were achieved for a fraction of the computational cost of the full system WF calculation while retaining high accuracy. The spin transition energies are of particular note as embedding can help break the functional dependency of calculations on transition metals. The absolutely localized Huzinaga WF-in-DFT method is an incredibly valuable tool for the chemical community and by publishing our code, user manual, and tutorial we seek to make the method as accessible as possible.

\section{Acknowledgement}

This research was carried out within the Nanoporous Materials Genome Center, which is supported by the U.S. Department of Energy, Office of Basic Energy Sciences, Division of Chemical Sciences, Geosciences, and Biosciences under Award DE-FG02-17ER16362. The authors acknowledge the Minnesota Supercomputing Institute (MSI) at the University of Minnesota and the National Energy Research Scientific Computing Center (NERSC), a DOE Office of Science User Facility supported by the Office of Science of the U.S. Department of Energy under Contract No. DE-AC02-05CH11231, for providing resources that contributed to the results reported within this paper.

\section{Supporting Information Available}

All relevant embedding output files, canonical output files, structure xyz geometry files, and DFT-in-DFT compared to KS-DFT energies for various subsystem sizes and charging methods is present in the Supporting Information. This information is available free of charge via the Internet at http://pubs.acs.org/. 


\section{References}

(1) Hohenberg, P.; Kohn, W. Inhomogeneous electron gas. Physical Review 1964, 136, B864-B871.

(2) Kohn, W.; Sham, L. J. Self-Consistent Equations Including Exchange and Correlation Effects. Physical Review 1965, 140, A1133-A1138.

(3) Siegbahn, P. E. M. The performance of hybrid DFT for mechanisms involving transition metal complexes in enzymes. JBIC Journal of Biological Inorganic Chemistry 2006, 11, 695-701.

(4) Quintal, M. M.; Karton, A.; Iron, M. A.; Boese, A. D.; Martin, J. M. L. Benchmark Study of DFT Functionals for Late-Transition-Metal Reactions $\dagger$. The Journal of Physical Chemistry A 2006, 110, 709-716.

(5) Minenkov, Y.; Occhipinti, G.; Jensen, V. R. MetalPhosphine Bond Strengths of the Transition Metals: A Challenge for DFT †. The Journal of Physical Chemistry A 2009, 113, 11833-11844.

(6) Moltved, K. A.; Kepp, K. P. The Metal Hydride Problem of Computational Chemistry: Origins and Consequences. The Journal of Physical Chemistry A 2019, 123, 2888-2900.

(7) Ghosh, A.; Taylor, P. R. High-level ab initio calculations on the energetics of low-lying spin states of biologically relevant transition metal complexes: a first progress report. Current Opinion in Chemical Biology 2003, 7, 113-124.

(8) Harvey, J. N. Structure and Bonding; Springer, Berlin, Heidelberg, 2004; Vol. 112; pp $151-184$.

(9) Ghosh, A. Transition metal spin state energetics and noninnocent systems: challenges for DFT in the bioinorganic arena. JBIC Journal of Biological Inorganic Chemistry 2006, 11, 712-724. 
(10) Hughes, T. F.; Friesner, R. A. Correcting Systematic Errors in DFT Spin-Splitting Energetics for Transition Metal Complexes. Journal of Chemical Theory and Computation 2011, r, 19-32.

(11) Lawson Daku, L. M.; Aquilante, F.; Robinson, T. W.; Hauser, A. Accurate Spin-State Energetics of Transition Metal Complexes. 1. CCSD(T), CASPT2, and DFT Study of $[\mathrm{M}(\mathrm{NCH}) 6] 2+(\mathrm{M}=\mathrm{Fe}, \mathrm{Co})$. Journal of Chemical Theory and Computation 2012, $8,4216-4231$.

(12) Radoń, M. Revisiting the role of exact exchange in DFT spin-state energetics of transition metal complexes. Phys. Chem. Chem. Phys. 2014, 16, 14479-14488.

(13) Radoń, M. Spin-State Energetics of Heme-Related Models from DFT and Coupled Cluster Calculations. Journal of Chemical Theory and Computation 2014, 10, 23062321.

(14) Cohen, A. J.; Mori-Sanchez, P.; Yang, W. Insights into Current Limitations of Density Functional Theory. Science 2008, 321, 792-794.

(15) Jones, R. O. Density functional theory: Its origins, rise to prominence, and future. Reviews of Modern Physics 2015, 87, 897-923.

(16) Yu, H. S.; Li, S. L.; Truhlar, D. G. Perspective: Kohn-Sham density functional theory descending a staircase. The Journal of Chemical Physics 2016, 145, 130901.

(17) Cohen, A. J.; Mori-Sánchez, P.; Yang, W. Challenges for Density Functional Theory. Chemical Reviews 2012, 112, 289-320.

(18) Wen, X.; Graham, D. S.; Chulhai, D. V.; Goodpaster, J. D. Absolutely Localized Projection-Based Embedding for Excited States. Journal of Chemical Theory and Computation 2020, 16, 385-398. 
(19) Warshel, A.; Levitt, M. Theoretical studies of enzymic reactions: Dielectric, electrostatic and steric stabilization of the carbonium ion in the reaction of lysozyme. Journal of Molecular Biology 1976, 103, 227-249.

(20) Svensson, M.; Humbel, S.; Froese, R. D. J.; Matsubara, T.; Sieber, S.; Morokuma, K. ONIOM: A Multilayered Integrated MO + MM Method for Geometry Optimizations and Single Point Energy Predictions. A Test for DielsAlder Reactions and Pt(P( t Bu) 3 ) 2 + H 2 Oxidative Addition. The Journal of Physical Chemistry 1996, 100, 19357-19363.

(21) Knizia, G.; Chan, G. K.-L. L. Density Matrix Embedding: A Strong-Coupling Quantum Embedding Theory. Journal of Chemical Theory and Computation 2013, 9, 1428-1432.

(22) Sun, Q.; Chan, G. K.-L. Quantum Embedding Theories. Accounts of Chemical Research 2016, 49, 2705-2712.

(23) Ding, F.; Manby, F. R.; Miller, T. F. Embedded Mean-Field Theory with BlockOrthogonalized Partitioning. Journal of Chemical Theory and Computation 2017, 13, 1605-1615.

(24) Ding, F.; Tsuchiya, T.; Manby, F. R.; Miller, T. F. Linear-Response Time-Dependent Embedded Mean-Field Theory. Journal of Chemical Theory and Computation 2017, 13, 4216-4227.

(25) Miyamoto, K.; Miller, T. F.; Manby, F. R. Fock-Matrix Corrections in Density Functional Theory and Use in Embedded Mean-Field Theory. Journal of Chemical Theory and Computation 2016, 12, 5811-5822.

(26) Fornace, M. E.; Lee, J.; Miyamoto, K.; Manby, F. R.; Miller, T. F. Embedded MeanField Theory. Journal of Chemical Theory and Computation 2015, 11, 568-580. 
(27) Fornace, M. E.; Lee, J.; Miyamoto, K.; Manby, F. R.; Miller, T. F. Correction to Embedded Mean-Field Theory. Journal of Chemical Theory and Computation 2015, 11, 3968-3968.

(28) Onida, G.; Reining, L.; Rubio, A. Electronic excitations: density-functional versus many-body Green's-function approaches. Reviews of Modern Physics 2002, 74, 601659.

(29) Chibani, W.; Ren, X.; Scheffler, M.; Rinke, P. Self-consistent Green's function embedding for advanced electronic structure methods based on a dynamical mean-field concept. Physical Review B 2016, 93, 165106.

(30) Rusakov, A. A.; Iskakov, S.; Tran, L. N.; Zgid, D. Self-Energy Embedding Theory (SEET) for Periodic Systems. Journal of Chemical Theory and Computation 2019, 15, $229-240$.

(31) Elliott, P.; Burke, K.; Cohen, M. H.; Wasserman, A. Partition density-functional theory. Physical Review A 2010, 82, 024501.

(32) Nafziger, J.; Wasserman, A. Density-Based Partitioning Methods for Ground-State Molecular Calculations. The Journal of Physical Chemistry A 2014, 118, 7623-7639.

(33) Gómez, S.; Nafziger, J.; Restrepo, A.; Wasserman, A. Partition-DFT on the water dimer. The Journal of Chemical Physics 2017, 146, 074106.

(34) Jacob, C. R.; Neugebauer, J. Subsystem density-functional theory. Wiley Interdisciplinary Reviews: Computational Molecular Science 2014, 4, 325-362.

(35) Lau, B. T. G.; Knizia, G.; Berkelbach, T. C. Regional Embedding Enables High-Level Quantum Chemistry for Surface Science. The Journal of Physical Chemistry Letters 2021, 12, 1104-1109. 
(36) Li, W.; Chen, M.; Rabani, E.; Baer, R.; Neuhauser, D. Stochastic embedding DFT: Theory and application to $\mathrm{p}$-nitroaniline in water. The Journal of Chemical Physics 2019, 151, 174115.

(37) Sushko, P. V.; Huang, C.; Govind, N.; Kowalski, K. Computational Materials Discovery; Royal Society of Chemistry, 2018; pp 87-116.

(38) Hedegård, E. D.; Reiher, M. Polarizable Embedding Density Matrix Renormalization Group. Journal of Chemical Theory and Computation 2016, 12, 4242-4253.

(39) Mühlbach, A. H.; Reiher, M. Quantum system partitioning at the single-particle level. The Journal of Chemical Physics 2018, 149, 184104.

(40) Hršak, D.; Olsen, J. M. H.; Kongsted, J. Polarizable Density Embedding Coupled Cluster Method. Journal of Chemical Theory and Computation 2018, 14, 1351-1360.

(41) Culpitt, T.; Brorsen, K. R.; Pak, M. V.; Hammes-Schiffer, S. Multicomponent density functional theory embedding formulation. The Journal of Chemical Physics 2016, 145, 044106.

(42) Nanda, K. D.; Krylov, A. I. The effect of polarizable environment on two-photon absorption cross sections characterized by the equation-of-motion coupled-cluster singles and doubles method combined with the effective fragment potential approach. The Journal of Chemical Physics 2018, 149, 164109.

(43) Ma, H.; Sheng, N.; Govoni, M.; Galli, G. Quantum Embedding Theory for Strongly Correlated States in Materials. Journal of Chemical Theory and Computation 2021, 17, 2116-2125.

(44) Manby, F. R.; Stella, M.; Goodpaster, J. D.; Miller, T. F. A Simple, Exact DensityFunctional-Theory Embedding Scheme. Journal of Chemical Theory and Computation 2012, 8, 2564-2568. 
(45) Chapovetsky, A.; Welborn, M.; Luna, J. M.; Haiges, R.; Miller, T. F.; Marinescu, S. C. Pendant Hydrogen-Bond Donors in Cobalt Catalysts Independently Enhance CO 2 Reduction. ACS Central Science 2018, 4, 397-404.

(46) Goodpaster, J. D.; Barnes, T. A.; Manby, F. R.; Miller, T. F. Density functional theory embedding for correlated wavefunctions: Improved methods for open-shell systems and transition metal complexes. The Journal of Chemical Physics 2012, 137, 224113.

(47) Goodpaster, J. D.; Barnes, T. A.; Manby, F. R.; Miller, T. F. Accurate and systematically improvable density functional theory embedding for correlated wavefunctions. The Journal of Chemical Physics 2014, 140, 18A507.

(48) Welborn, M.; Manby, F. R.; Miller, T. F. Even-handed subsystem selection in projection-based embedding. The Journal of Chemical Physics 2018, 149, 144101.

(49) Lee, S. J. R.; Ding, F.; Manby, F. R.; Miller, T. F. Analytical gradients for projectionbased wavefunction-in-DFT embedding. The Journal of Chemical Physics 2019, 151, 064112 .

(50) Claudino, D.; Mayhall, N. J. Simple and Efficient Truncation of Virtual Spaces in Embedded Wave Functions via Concentric Localization. Journal of Chemical Theory and Computation 2019, 15, 6085-6096.

(51) Claudino, D.; Mayhall, N. J. Automatic Partition of Orbital Spaces Based on Singular Value Decomposition in the Context of Embedding Theories. Journal of Chemical Theory and Computation 2019, 15, 1053-1064.

(52) Hégely, B.; Nagy, P. R.; Ferenczy, G. G.; Kállay, M. Exact density functional and wave function embedding schemes based on orbital localization. The Journal of Chemical Physics 2016, 145, 064107. 
(53) Bensberg, M.; Neugebauer, J. Orbital alignment for accurate projection-based embedding calculations along reaction paths. Journal of Chemical Theory and Computation 2020, 16, 3607-3619.

(54) Bensberg, M.; Neugebauer, J. Automatic basis-set adaptation in projection-based embedding. The Journal of Chemical Physics 2019, 150, 184104.

(55) Bensberg, M.; Neugebauer, J. Density functional theory based embedding approaches for transition-metal complexes †. Phys. Chem. Chem. Phys 2020, 22, 26093.

(56) Chulhai, D. V.; Goodpaster, J. D. Improved Accuracy and Efficiency in Quantum Embedding through Absolute Localization. Journal of Chemical Theory and Computation 2017, 13, 1503-1508.

(57) Graham, D. S.; Wen, X.; Chulhai, D. V.; Goodpaster, J. D. Robust, Accurate, and Efficient: Quantum Embedding Using the Huzinaga Level-Shift Projection Operator for Complex Systems. Journal of Chemical Theory and Computation 2020, 16, 22842295.

(58) Chulhai, D. V.; Goodpaster, J. D. Projection-Based Correlated Wave Function in Density Functional Theory Embedding for Periodic Systems. Journal of Chemical Theory and Computation 2018, 14, 1928-1942.

(59) Frisch, M. J.; Trucks, G. W.; Schlegel, H. B.; Scuseria, G. E.; Robb, M. A.; Cheeseman, J. R.; Scalmani, G.; Barone, V.; Petersson, G. A.; Nakatsuji, H.; Li, X.; Caricato, M.; Marenich, A. V.; Bloino, J.; Janesko, B. G.; Gomperts, R.; Mennucci, B.; Hratchian, H. P.; Ortiz, J. V.; Izmaylov, A. F.; Sonnenberg, J. L.; WilliamsYoung, D.; Ding, F.; Lipparini, F.; Egidi, F.; Goings, J.; Peng, B.; Petrone, A.; Henderson, T.; Ranasinghe, D.; Zakrzewski, V. G.; Gao, J.; Rega, N.; Zheng, G.; Liang, W.; Hada, M.; Ehara, M.; Toyota, K.; Fukuda, R.; Hasegawa, J.; Ishida, M.; Nakajima, T.; Honda, Y.; Kitao, O.; Nakai, H.; Vreven, T.; Throssell, K.; Montgomery Jr., J. A.; 
Peralta, J. E.; Ogliaro, F.; Bearpark, M. J.; Heyd, J. J.; Brothers, E. N.; Kudin, K. N.; Staroverov, V. N.; Keith, T. A.; Kobayashi, R.; Normand, J.; Raghavachari, K.; Rendell, A. P.; Burant, J. C.; Iyengar, S. S.; Tomasi, J.; Cossi, M.; Millam, J. M.; Klene, M.; Adamo, C.; Cammi, R.; Ochterski, J. W.; Martin, R. L.; Morokuma, K.; Farkas, O.; Foresman, J. B.; Fox, D. J. Gaussian16 Revision A.03. 2016.

(60) Zhao, Y.; Truhlar, D. G. The M06 suite of density functionals for main group thermochemistry, thermochemical kinetics, noncovalent interactions, excited states, and transition elements: two new functionals and systematic testing of four M06-class functionals and 12 other function. Theoretical Chemistry Accounts 2008, 120, 215-241.

(61) Dunning, T. H. Gaussian basis sets for use in correlated molecular calculations. I. The atoms boron through neon and hydrogen. The Journal of Chemical Physics 1989, 90, $1007-1023$.

(62) Lee, K.; Isley, W. C.; Dzubak, A. L.; Verma, P.; Stoneburner, S. J.; Lin, L.-C.; Howe, J. D.; Bloch, E. D.; Reed, D. A.; Hudson, M. R.; Brown, C. M.; Long, J. R.; Neaton, J. B.; Smit, B.; Cramer, C. J.; Truhlar, D. G.; Gagliardi, L. Design of a Metal-Organic Framework with Enhanced Back Bonding for Separation of N 2 and CH 4. Journal of the American Chemical Society 2014, 136, 698-704.

(63) Radon, M. R. Benchmarking quantum chemistry methods for spin-state energetics of iron complexes against quantitative experimental data †. Phys. Chem. Chem. Phys $2019,4854,4854$.

(64) Sun, Q.; Berkelbach, T. C.; Blunt, N. S.; Booth, G. H.; Guo, S.; Li, Z.; Liu, J.; McClain, J. D.; Sayfutyarova, E. R.; Sharma, S.; Wouters, S.; Chan, G. K.-L. PySCF: the Python-based simulations of chemistry framework. Wiley Interdisciplinary Reviews: Computational Molecular Science 2018, 8, e1340.

(65) Werner, H.-J.; Knowles, P. J.; Knizia, G.; Manby, F. R.; Schütz, M. Molpro: a general- 
purpose quantum chemistry program package. Wiley Interdisciplinary Reviews: Computational Molecular Science 2012, 2, 242-253.

(66) Werner, H.-J.; Knowles, P. J.; Knizia, G.; Manby, F. R.; Schütz, M.; Celani, P.; Györffy, W.; Kats, D.; Korona, T.; Lindh, R.; Mitrushenkov, A.; Rauhut, G.; Shamasundar, K. R.; Adler, T. B.; Amos, R. D.; Bennie, S. J.; Bernhardsson, A.; Berning, A.; Cooper, D. L.; Deegan, M. J. O.; Dobbyn, A. J.; Eckert, F.; Goll, E.; Hampel, C.; Hesselmann, A.; Hetzer, G.; Hrenar, T.; Jansen, G.; Köppl, C.; Lee, S. J. R.; Liu, Y.; Lloyd, A. W.; Ma, Q.; Mata, R. A.; May, A. J.; McNicholas, S. J.; Meyer, W.; Miller III, T. F.; Mura, M. E.; Nicklass, A.; O’Neill, D. P.; Palmieri, P.; Peng, D.; Pflüger, K.; Pitzer, R.; Reiher, M.; Shiozaki, T.; Stoll, H.; Stone, A. J.; Tarroni, R.; Thorsteinsson, T.; Wang, M.; Welborn, M. MOLPRO, version 2019.2, a package of ab initio programs. 2019.

(67) Győrffy, W.; Shiozaki, T.; Knizia, G.; Werner, H.-J. Analytical energy gradients for second-order multireference perturbation theory using density fitting. The Journal of Chemical Physics 2013, 138, 104104.

(68) Shiozaki, T.; Győrffy, W.; Celani, P.; Werner, H.-J. Communication: Extended multistate complete active space second-order perturbation theory: Energy and nuclear gradients. The Journal of Chemical Physics 2011, 135, 081106.

(69) Celani, P.; Werner, H.-J. Multireference perturbation theory for large restricted and selected active space reference wave functions. The Journal of Chemical Physics 2000, 112, 5546-5557.

(70) Knowles, P. J.; Hampel, C.; Werner, H. Coupled cluster theory for high spin, open shell reference wave functions. The Journal of Chemical Physics 1993, 99, 5219-5227.

(71) Knowles, P. J.; Hampel, C.; Werner, H.-J. Erratum: "Coupled cluster theory for high 
spin, open shell reference wave functions" [ J. Chem. Phys. 99 , 5219 (1993)]. The Journal of Chemical Physics 2000, 112, 3106-3107.

(72) Goodpaster, J. D.; Graham, D. S.; Chulhai, D. V.; Xuelan, W. Goodpaster/QSoME: Open-shell Embedding. 2021; https://github.com/Goodpaster/QSoME.

(73) Cirera, J.; Via-Nadal, M.; Ruiz, E. Benchmarking Density Functional Methods for Calculation of State Energies of First Row Spin-Crossover Molecules. Inorganic Chemistry 2018, 57, 14097-14105. 


\section{Graphical TOC Entry}

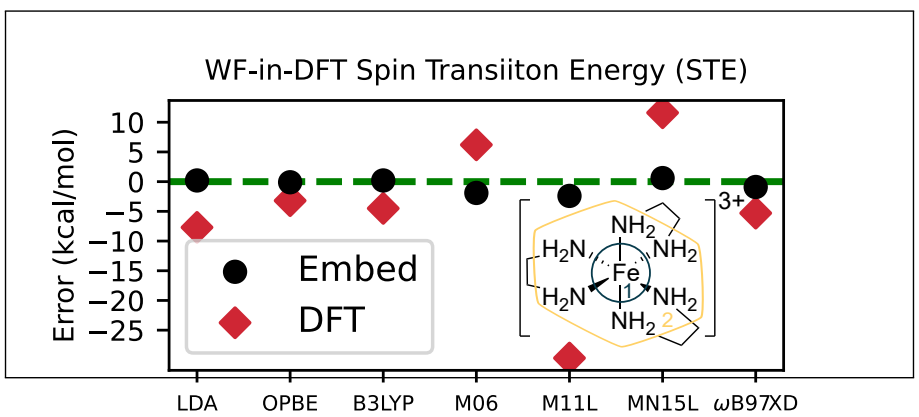

